\title{
SOME CLASSES OF E-COMPACTNESS
}

\author{
ROBERT L. BLEFKO \\ (Received 10 September 1969) \\ Communicated by J.P. O. Silberstein
}

\section{Introduction}

Mrowka and Engleking [1] have recently introduced a notion more general than that of compactness. Perhaps the most convenient direction at departure is the following: for spaces $X$ and $E, X$ is said to be $E$-compact if $X$ is topologically embeddable as a closed subset of a product $E^{m}$ for some cardinal $m$, in which case we write $X \subset_{c l} E^{m}$. More generally, $X$ is said to be $E$-completely regular if $X$ is topologically embeddable in a product $E^{m}$ for some $m$. For example, if we take $E$ to be the unit interval $I$, we obtain the class of compact spaces and completely regular spaces, respectively, as is well-known. The question then arises, of course, given a space $E$, what spaces are compact with respect to it? A related question, to which we address ourselves in this note, is the following. Denote by $K[E]$ all those topological spaces which are $E$-compact. Then we ask: are there very many distinct $E$-compact classes? It will develop that there are indeed quite a large number of such classes.

There are several results, some of which are almost obvious, which we will need and can be found discussed in [2].

a) For any $E$-completely regular space there exists an extension $\beta_{E} X$, containing $X$ densely, which has the following properties:

(i) $\beta_{E} X$ is $E$-compact

(ii) Each continuous $f: X \rightarrow E$ admits a continuous extension $f^{*}: \beta_{E} X \rightarrow E$

(iii) If $Y$ is an $E$-compact space, then any continuous $f: X \rightarrow Y$ admits a continuous extension $f^{*}: \beta_{E} X \rightarrow Y$

(iv) The extension $\beta_{E} X$ is unique in the sense that if $T$ is any extension of $X$ satisfying (i)-(ii) there is a homeomorphism $\phi$ of $\beta_{E} X$ onto $T$ such that $\phi$ is the identity on $X$.

b) Let $I, R, \subseteq$ and $N$ denote the closed unit interval, the reals, the two-point discrete space, and the natural numbers, respectively; we then have

(i) $X$ is $I$-compact iff $X$ is compact

(ii) $X$ is $R$-compact iff $X$ is realcompact 
(iii) $X$ is $\mathfrak{D}$-compact iff $X$ is 0 -dimensional (in the sense that there exists a base of open-closed sets) and compact

(iv) if $X$ is $N$-compact, then $X$ is 0 -dimensional and realcompact

c) An arbitrary product of $E$-compact spaces is $E$-compact

d) A closed subset of an $E$-compact space is $E$-compact

e) If $E_{1} \in K\left[E_{2}\right]$ then $K\left[E_{1}\right] \subset K\left[E_{2}\right]$

f) $K\left[E_{1}\right]=K\left[E_{2}\right]$ iff $E_{1}$ is $E_{2}$-compact and $E_{2}$ is $E_{1}$-compact

g) Let $E$ be $E_{1}$-completely regular and vice versa, and let a space $X$ be $E$ completely regular. Then $\beta_{E} X$ can be characterized as the set of all points in $\beta_{E_{1}} X-X_{0}$ where $X_{0}$ is the set of all points $p_{0}$ of $\beta_{E_{1}} X$ such that there is a continuous $f: \beta_{E_{1}} X \rightarrow Y, Y$ any $E_{1}$-compact space containing $E$, with $f(p) \in E$ for $p \in X$ and $f\left(p_{0}\right) \in Y-E$.

We will call this last stated result Mrowka's Theorem ([2], Theorem 4.18); it will find frequent use in this paper.

Our remarks will deal mostly with subspaces of the ordinals (with the order topology) and spaces derived from them; they provide a particularly fertile area for examples in this connection. By $S(\alpha)$ we mean all ordinals less than $\alpha ; \omega_{\alpha}$ will be used for the smallest ordinal of cardinality $\aleph_{\alpha}$; a limit ordinal is one with no immediate predecessor. We also introduce some spaces related to segments of the ordinals. Let $\alpha$ be any non-limit ordinal greater than 1 and consider the space $S\left(\omega_{\alpha}\right)$. We will denote by $E_{\alpha}$ the 'generalized leng line', i.e., the space obtained by using $S\left(\omega_{\alpha}\right)$ instead of $S\left(\omega_{1}\right)$ in the construction of the 'long line' (See [3], p. 55). The following facts about $E_{\alpha}$ (and respectively, $S\left(\omega_{\alpha}\right)$ ) hold:

1. Of any two disjoint closed sets in $E_{\alpha}$ (resp., $S\left(\omega_{\alpha}\right)$ ), one is bounded by some element of $E_{\alpha}$ (resp., $S\left(\omega_{\alpha}\right)$ ).

2. Every continuous $f: E_{\alpha} \rightarrow R$ (resp., $f: S\left(\omega_{\alpha}\right) \rightarrow R$ ) is constant on a tail.

3. Any subset of $E_{\alpha}$ (resp., $S\left(\omega_{\alpha}\right)$ ) of cardinality less than $\aleph_{\alpha}$ is bounded.

4. $E_{\alpha}$ (resp. $S\left(\omega_{\alpha}\right)$ ) is countably compact and hence also pseudocompact.

We now proceed to the main results.

\section{2}

Let $\alpha$ be any limit ordinal less than $\omega_{1}$. Then neither of $S(\alpha), S\left(\omega_{1}\right)$ is compact with respect to the other.

Proof. We first need to show that $\left[S\left(\omega_{1}\right)\right]^{m}$ is countably compact for any cardinal $m$. To show this, let $A$ be any countable subset contained in $\left[S\left(\omega_{1}\right)\right]^{m}$. Then in each coordinate space $S\left(\omega_{1}\right)_{\xi}, \pi_{\xi}[A]$ is countable and hence it must be 
bounded by, say, $\eta_{\xi}$; that is, $x \leqq \eta_{\xi}$ for each $x$ in $\pi_{\xi}[A]$. But then $A$ is contained as a subset of the product $\prod_{\xi} S\left(\eta_{\xi}+1\right)$ which is compact since each of the factor spaces is compact. Thus, $A$ has a limit in this subspace of $\left[S\left(\omega_{1}\right)\right]^{m}$ and hence in the whole product also. It follows that $\left[S\left(\omega_{1}\right)\right]^{m}$ is sequentially compact and therefore countably compact.

(i) To show $S(\alpha)$ is not $S\left(\omega_{1}\right)$-compact, we first note that there exists a countable sequence $\left\{p_{n}\right\}$ of ordinals less than $\alpha$ that has no limit in $S(\alpha)$. So, if $S(\alpha)$ were embeddable in $\left[S\left(\omega_{1}\right)\right]^{m}$ for some $m$ as a closed copy, then, since $\left[S\left(\omega_{1}\right)\right]^{m}$ is countably compact, the sequence $\left\{p_{n}\right\}$ in the copy of $S(\alpha)$ would have a limit and this limit would be in $S(\alpha)$, being closed; this is a contradiction.

(ii) $S\left(\omega_{1}\right)$ is not $S(\alpha)$-compact: first, recall that $S\left(\omega_{1}\right)$ is pseudocompact but $S(\alpha)$ is not. Now, if $S\left(\omega_{1}\right)$ were contained as a closed copy in $[S(\alpha)]^{m}=$ $\prod_{\xi} S(\alpha)_{\xi}\left(S(\alpha)_{\xi}=S(\alpha)\right.$ for each $\left.\xi \in \Xi\right)$ then $\pi_{\xi}\left[S\left(\omega_{1}\right)\right]$ must be unbounded in some $S(\alpha)_{\xi}$ for if not, then $S\left(\omega_{1}\right)$ would be contained as a closed copy of compact spaces and hence would be compact. Let this coordinate space by $S(\alpha)_{\xi_{0}}$. Consider any sequence $1=x_{1}, x_{2}, \cdots$ of isolated points in $S(\alpha)$ such that $x_{n} \rightarrow \alpha$. Then the sets

$$
\left\{U_{n}=\left\{p \in S(\alpha): x_{n} \leqq p<x_{n+1}\right\}: n \in N\right\}
$$

partition $S(\alpha)_{\xi_{0}}$ into open-closed sets from which we see that the collection $\left\{V_{n}=\pi_{\xi}^{-1}\left[U_{n}\right]\right\}$ is a countably infinite partition of $\prod_{\xi} S(\alpha)_{\xi}$. Define $f: \prod_{\xi} S(\alpha)_{\xi} \rightarrow N$, the set of natural numbers, by $f\left[V_{n}\right]=n$. Then $f$ is continuous and the restriction to the copy of $S\left(\omega_{1}\right)$ is continuous and unbounded, which cannot be, since unbounded continuous real-valued functions on $S\left(\omega_{1}\right)$ do not exist. [Alternately, cf. [4], p. 20].

If $\alpha, \beta$ are distinct non-limit ordinals and $\omega_{\alpha}, \omega_{\beta}$ are the smallest ordinals of cardinality $\boldsymbol{\aleph}_{\alpha}, \boldsymbol{\aleph}_{\beta}$ respectively, then neither of $S\left(\omega_{\alpha}\right), S\left(\omega_{\beta}\right)$ is compact with respect to the other.

Proof. For definiteness let $\omega_{\alpha}<\omega_{\beta}$.

(i) $S\left(\omega_{\alpha}\right)$ is not $S\left(\omega_{\beta}\right)$-compact: for suppose that for some cardinal $m$, $S\left(\omega_{\alpha}\right) \subset_{c l}\left[S\left(\omega_{\beta}\right)\right]^{m}$. Let $\left\{p_{\xi}\right\}$ be a net in $S\left(\omega_{\alpha}\right)$ such that $p_{\xi} \rightarrow \omega_{\alpha}$. Then $\left|\left\{p_{\xi}\right\}\right| \leqq \boldsymbol{N}_{\alpha}$. Now the projections of $\left\{p_{\xi}\right\}$ on each of the coordinate spaces $S\left(\omega_{\beta}\right)_{\lambda}$ are of cardinality $\leqq \boldsymbol{\aleph}_{\alpha}<\boldsymbol{\aleph}_{\beta}$ so there exists a sup in $S\left(\omega_{\beta}\right)_{\lambda}$ for each of these projections; say $\pi_{\lambda}\left(p_{\xi}\right) \rightarrow x_{\lambda}$ in the $\lambda$-th coordinate space $S\left(\omega_{\beta}\right)_{\lambda}$. But then $\left\{p_{\xi}\right\}$ has as limit the point $\left(x_{\lambda}\right) \in \prod_{\lambda} S\left(\omega_{\beta}\right)_{\lambda}$ and this limit must be in the copy of $S\left(\omega_{\alpha}\right)$ since it is closed. This is contrary to the assumption.

(ii) To show that $S\left(\omega_{\beta}\right)$ is not $S\left(\omega_{\alpha}\right)$-compact, first observe that $\beta S\left(\omega_{\beta}\right)=$ $S\left(\omega_{\beta}\right) \cup\left\{\omega_{\beta}\right\}$ and likewise for the space $\beta S\left(\omega_{\alpha}\right)$. (Cf. [4] p. 137). We intend to show that there exist no continuous $f: \beta S\left(\omega_{\beta}\right) \rightarrow S\left(\omega_{\alpha}\right)$ such that $f\left(\omega_{\beta}\right)=\omega_{\alpha}$ and 
$f(p)<\omega_{\alpha}$ for all $p \in S\left(\omega_{\beta}\right)$ and then use Mrowka's Theorem with $X=S\left(\omega_{\beta}\right)$, $E=S\left(\omega_{\alpha}\right), E_{1}=I, Y=\beta S\left(\omega_{\alpha}\right)$, and $\beta_{E_{1}} X=\beta S\left(\omega_{\beta}\right)$. Assume there is such a function. There are two cases to check.

a) Suppose for every $a \in S\left(\omega_{\alpha}\right), f^{-1}(a)$ is bounded in $S\left(\omega_{\beta}\right)$. Then for every $a \in S\left(\omega_{\alpha}\right)$ there exists an ordinal $\eta_{a}=\sup f^{-1}(a)$. Then the set $\left\{\eta_{a}: a \in S\left(\omega_{\alpha}\right)\right\}$, being of cardinality $\leqq \boldsymbol{\aleph}_{\alpha}$, has a sup in $S\left(\omega_{\beta}\right)$, say it is $x_{0}<\omega_{\beta}$. But then $f$ is not defined for $p>x_{0}$ which makes no sense.

b) Thus, there must be an $a_{0} \in S\left(\omega_{\alpha}\right)$ such that $f^{-1}\left(a_{0}\right)$ is unbounded in $S\left(\omega_{\beta}\right)$ and there can be only one such since $S\left(\omega_{\beta}\right)$ cannot have two disjoint closed unbounded subsets. Now if the set of points in $S\left(\omega_{\beta}\right)$ with image values different from $a_{0}$ under $f$ is cofinal in $S\left(\omega_{\beta}\right)$, let $\left\{x_{a}\right\}$ be this set of images. Then for each $x_{a}, f^{-1}\left(x_{a}\right)$ has a sup, say $\eta_{a}$, less than $\omega_{\beta}$ and $\sup \left\{\eta_{a}\right\}<\omega_{\beta}$ since $\left|\left\{\eta_{a}\right\}\right| \leqq \boldsymbol{\aleph}_{\alpha}$. Hence, on a tail of $S\left(\omega_{\beta}\right), f(p)=a_{0}$ so that $f\left(\omega_{\beta}\right)=a_{0}$ and not $\omega_{\alpha}$ which is a contradiction.

We now consider ordinals which are not of the form $\omega_{\alpha}$ where $\alpha$ is non-limit. At this point we pause to introduce some terminology which is mostly that of Sierpinski [5]. We divide all ordinals into classes, putting two ordinals in the same class if they are of the same cardinality. The least number in such a class is called an initial ordinal. In our previous notation, e.g., $\omega_{\alpha}$, the least ordinal of cardinality $\boldsymbol{\aleph}_{\alpha}$, is an initial ordinal. Initial ordinals $\omega_{\alpha}$ which are limits of transfinite sequences of type $<\omega_{\alpha}$ are named singular; all other initial numbers, i.e., ordinals $\omega_{\alpha}$ which are not the limits of transfinite sequences of type $<\omega_{a}$ are called regular. We emphasize that the terms singular and regular apply only to initial numbers. We say $\alpha$ is cofinal (some authors say confinal) with $\beta \leqq \alpha$ if there exists a strictly increasing sequence of order type $\beta$ with $\alpha$ as a limit. In order that an initial ordinal $\omega_{\alpha}$ be singular it is necessary and sufficient that it be cofinal with a certain number of the second kind (i.e., a limit ordinal) smaller than $\omega_{\alpha}$ [5]. We then define two ordinals $\alpha$ and $\beta$ to be of the same degree of cofinality, in symbols, $\operatorname{cf}(\alpha)=\operatorname{cf}(\beta)$, if there exists an ordinal $\eta$ such that $\alpha$ and $\beta$ are cofinal with $\eta$ and $\eta$ is the smallest such ordinal for $\alpha$ and $\beta$ both. It perhaps should be mentioned that there always is, for any ordinal $\alpha$, a smallest ordinal $\gamma$ such that $\alpha$ is cofinal with $\gamma$. This follows from the facts that the ordinals are well-ordered and that if $\alpha$ is cofinal with $\beta$ and $\beta$ is cofinal with $\gamma$ then $\alpha$ is cofinal with $\gamma$. In addition, we note that this smallest ordinal must be regular. (See [6], p. 27.)

EXAMPLeS. $\operatorname{cf}\left(\omega_{0}\right)=\operatorname{cf}\left(\omega_{\omega_{0}}\right)=\operatorname{cf}(a)$ where $\omega_{a}$ is the smallest ordinal which satisfies the equation $\omega_{a}=a$. (See [5], p. 407 or [7].) We can now state the

THEOREM. For initial ordinals $\alpha, \beta, K[S(\alpha)]=K[S(\beta)]$ if and only if $\mathrm{cf}(\alpha)=$ $\operatorname{cf}(\beta)$. 
Proof. (Sufficiency) Let $\eta$ be the smallest ordinal with which both $\alpha$ and $\beta$ are cofinal. We will show that $K[S(\alpha)]=K[S(\eta)]$. That $K[S(\beta)])=K[S(\eta)]$ follows in the same way.

We assume that $\eta<\alpha$ of course, so that there is something to prove. Now there exists a sequence $\left\{p_{\xi}\right\}_{\xi<\eta}$ which is strictly increasing and has $\alpha$ for a limit. The function $f: S(\eta) \rightarrow S(\alpha)$ given by $f(\xi)=p_{\xi}$ may not be continuous but we can alter it to a function $\phi$ which is continuous as follows: if $p$ is isolated then $\phi(p)=f(p)$; if $p$ is not isolated than $\phi(p)=\sup \{f(x): x<p\}$. Then $\phi$ is continuous, strictly increasing, and its range is still unbounded in $S(\alpha)$ since the set of image values of isolated points under $f$ is unbounded in $S(\alpha)$. The function $\phi$ can be continuously extended to $\phi^{*}: \beta S(\eta) \rightarrow \beta S(\alpha)$ by setting $\phi^{*}(\eta)=\alpha$. We then use Mrowka's Theorem again to show that $S(\eta)$ is $S(\alpha)$-compact.

Next, we want a continuous function $\psi$ of the right kind from $\beta S(\alpha)$ to $\beta S(\eta)$; that is, $\psi$ should be such that $\psi(p) \in S(\eta)$ for $p \in S(\alpha)$ and $\psi(\alpha)=\eta$. If $p \in S(\alpha)$ is an image of the function $\phi$ above, we define $\psi(p)=\phi^{-1}(p)$. If $p \in S(\alpha)$ is not an image, then the set of all images of $\phi$ which are greater than $p$ has a least element $p_{2}$. Also, the set of all images of $\phi$ which are less than $p$ has a greatest element $p_{1}$. This follows from the fact that $\phi$ is continuous and strictly increasing. Then for all $p \in S(\alpha)$ such that $p_{1}<p<p_{2}$, define $\psi(p)=\phi^{-1}\left(p_{2}\right)$. In addition, define $\psi(\alpha)=\eta$. Then $\psi$ is of the required sort.

From the foregoing, we see that $K[S(\alpha)]=K[S(\eta)]=K[S(\beta)]$. (Cf. Introduction, item $f$ ).)

(Necessity). Let $\operatorname{cf}(\alpha) \neq \operatorname{cf}(\beta)$. We want to conclude that neither of $S(\alpha)$, $S(\beta)$ is compact with respect to the other.

Let $\alpha^{\prime}$ and $\beta^{\prime}$ be the smallest ordinals with which $\alpha$ and $\beta$, resp., are cofinal. Then as in the immediately preceding discussion, $K[S(\alpha)]=K\left[S\left(\alpha^{\prime}\right)\right]$ and also $K[S(\beta)]=K\left[S\left(\beta^{\prime}\right)\right]$. We will show that neither of $S\left(\alpha^{\prime}\right), S\left(\beta^{\prime}\right)$ is compact with respect to the other. Suppose for definiteness that $\alpha^{\prime}<\beta^{\prime}$.

(i) First note that the ordinals $\alpha^{\prime}$ and $\beta^{\prime}$ are regular. Then $S\left(\alpha^{\prime}\right)$ is not $S\left(\beta^{\prime}\right)$ compact for the reason that there can exist no continuous function $f$ from $\beta S\left(\alpha^{\prime}\right)=$ $S\left(\alpha^{\prime}\right) \cup\left\{\alpha^{\prime}\right\}$ to $\beta S\left(\beta^{\prime}\right)=S\left(\beta^{\prime}\right) \cup\left\{\beta^{\prime}\right\}$, using the regularity of the oridinal $\beta^{\prime}$.

(ii) That $S\left(\beta^{\prime}\right)$ is not $S\left(\alpha^{\prime}\right)$-compact is shown by an argument which is entirely analogous to the proof given in (ii) of Section $\mathbf{C}$.

\section{5.}

For arbitrary limit ordinals $\alpha, \beta$ both $S(\alpha)$ and $S(\beta)$ may be compact with respect to each other.

EXAMPLE. Consider the ordinals $\omega_{0}$ and $\omega_{0}+\omega_{0}$. Clearly $S\left(\omega_{0}\right)$ is $S\left(\omega_{0}+\omega_{0}\right)$ compact since it is homeomorphic to the closed set $\left\{p: p>\omega_{0}\right\}$ in $S\left(\omega_{0}+\omega_{0}\right)$. Next, consider the function $f: \beta S\left(\omega_{0}+\omega_{0}\right) \rightarrow \beta S\left(\omega_{0}\right)$ given by 


$$
\begin{array}{ll}
f(p)=1 & \text { for } p \leqq \omega_{0}+1 \\
f\left(\omega_{0}+n\right)=n & \text { for } \mathrm{n} \geqq 2 \\
f\left(\omega_{0}+\omega_{0}\right)=\omega_{0} . &
\end{array}
$$

We now utilize Mrowka's Theorem again, letting $E=S\left(\omega_{0}\right), E_{1}=[0,1]$, $Y=S\left(\omega_{0}\right) \cup\left\{\omega_{0}\right\}=\beta S\left(\omega_{0}\right)$, and $X=S\left(\omega_{0}+\omega_{0}\right)$. Then it is seen that $\beta_{E_{1}} X=$ $\beta S\left(\omega_{0}+\omega_{0}\right)$. Now the quoted result states that $\beta_{E} X=\beta_{S\left(\omega_{0}\right)} S\left(\omega_{0}+\omega_{0}\right)$ is the set of all points of $\beta_{E_{1}} X-X_{0}$ where $X_{0}$ is the set of all points $p$ such that there is an $f: \beta S\left(\omega_{0}+\omega_{0}\right) \rightarrow \beta S\left(\omega_{0}\right)$ such that $f(p) \in S\left(\omega_{0}\right)$ for $p \in S\left(\omega_{0}+\omega_{0}\right)$ and $f\left(\omega_{0}+\omega_{0}\right)=\omega_{0}$. The function defined above is of the right kind, and thus, since $X \subset \beta_{E} X$ always, $X$ must be $\beta_{E} X$. That is,

$$
S\left(\omega_{0}+\omega_{0}\right)=\beta_{S\left(\omega_{0}\right)} S\left(\omega_{0}+\omega_{0}\right)
$$

which means that $S\left(\omega_{0}+\omega_{0}\right)$ is $S\left(\omega_{0}\right)$-compact.

REMARK. Even if the cardinality of $\alpha$ and $\beta$ are not equal we may still have $S(\alpha)$ and $S(\beta)$ of the same degree of compactness. For, consider the ordinals $\omega_{0}$ and $\omega_{1}+\omega_{0}$. Then by an analogous procedure to the above, we may show that $K\left[S\left(\omega_{0}\right)\right]=K\left[S\left(\omega_{1}+\omega_{0}\right)\right]$.

We now consider the "generalized long lines" $E_{\alpha}$ mentioned in the Introduction. Then for any non-limit ordinals $\alpha$ and $\beta$, neither of $E_{\alpha}, E_{\beta}$ is compact with respect to the other. The proof is analogous to a former proof. In fact, if $\alpha<\beta$, to show $E_{\alpha}$ is not $E_{\beta}$-compact, we proceed as in (i) of Section 3. To show $E_{\beta}$ is not $E_{\alpha}$-compact, we argue is in (ii) of Section 3. Note that none of the classes $K\left[E_{\alpha}\right]$ is equal to any of the classes $K[S(\alpha)]$. This follows from the fact that for any $\alpha$, $S(\alpha)$ is 0 -dimensional (in the sense that there exists a base of open-closed sets) whereas none of the $E_{\alpha}$ is.

In the discussion of the long lines there is one additional question to settle: if $E_{0}$ is taken to be the reals, which is natural, how do $K\left[E_{0}\right]$ and $K\left[E_{\alpha}\right]$ compare? Again, neither is compact with respect to the other as we now show.

(i) $E_{0}$ is not $E_{\alpha}$-compact: first recall that $E_{\alpha}$ is countably compact as is $\left(E_{\alpha}\right)^{m}$ for any cardinal $m$. Thus, if $E_{0}$ were embeddable as a closed subset of $\left(E_{\alpha}\right)^{m}$ for some $m$ then $E_{0}$, being a closed subset of countably compact space, would be countably compact which is not true.

(ii) $E_{\alpha}$ is not $E_{0}$-compact (that is, realcompact). This follows from the fact that $E_{\alpha}$ contains a closed copy of $S\left(\omega_{\alpha}\right)$ which is not realcompact. Then if $E_{\alpha} \subset_{c l}$ $\left(E_{0}\right)^{m}$ for some $m, S\left(\omega_{\alpha}\right)$ would be contained as a closed copy also in $\left(E_{0}\right)^{m}$ which cannot be. 
Lastly, we construct a space $Z$ which is interesting just to look at and whose inclusion here will settle certain questions which arose in connection with the previous material. First, it will lead the way towards many more classes of compactness and also, it will answer the question in the negative whether the classes $K\left[S\left(\omega_{\alpha}\right)\right]$, for $\alpha>0$ and non-limit, are atoms in the lattice of classes of $E$-compact spaces (as was suspected originally). The reason for this suspicion is as follows: for the two-point discrete space $\mathfrak{D}$ and the space $N$ of natural numbers, there is no class between the 'bottom' $K(\mathfrak{D})$ and $K[N]=K\left[S\left(\omega_{0}\right)\right]$, as Mrowka has shown [2]. Thus the possibility existed that the classes $K\left[S\left(\omega_{\alpha}\right)\right]$ for $\alpha>0$ were also atoms, which would have been a pleasant situation. The space $Z$ to be constructed will be in a class between $K[\mathfrak{D}]$ and $K\left[S\left(\omega_{1}\right)\right]$.

The space $Z$. The underlying space is $S\left(\omega_{1}\right)$. For any ordinal $\alpha<\omega_{0}$ we let $T_{\alpha}=S(\alpha)$ and $T_{\alpha}^{*}=S(\alpha+1)$. We also let $T_{\omega_{0}}=\bigcup_{\alpha<\omega_{0}} T_{\alpha}^{*}$ with the following topology: $U$ is open in $T_{\omega_{0}}$ iff $U \cap T_{\alpha}^{*}$ is open in $T_{\alpha}^{*}$ for all $\alpha<\omega_{0}$. We then define $T_{\omega_{0}}^{*}=\beta T_{\omega_{0}}$. [Note that $T_{\omega_{0}}$ is simply $N$ so that $\beta T_{\omega_{0}}=T_{\omega_{0}}^{*}=\beta N$.]

Now suppose that $T_{\alpha}^{*}$ has been defined for all ordinals $<$ some $\xi$, that for $\alpha<\gamma<\xi, T_{\alpha}^{*} \subset T_{\gamma}^{*}$. On the union $T_{\xi}=\bigcup_{\alpha<\xi} T_{\alpha}^{*}$ is put the following topology: $U$ is open in $T_{\xi}$ iff $U \cap T_{\alpha}^{*}$ is open for all $\alpha<\xi$. We then define $T_{\xi}^{*}=\beta T_{\xi}$.

Note that $T_{\xi}^{*}$ contains a copy of the set of all isolated ordinals $\leqq \xi$ and that this copy, which is countable, is dense in $T_{\xi}$. We conclude that $T_{\xi}^{*}=\beta T_{\xi}=\beta N$.

We then define $Z=\bigcup_{\alpha<\omega_{1}} T_{\alpha}^{*}$ with the following topology: $U$ is open in $Z$ iff $U \cap T_{\alpha}^{*}$ is open in $T_{\alpha}^{*}$ for every $\alpha<\omega_{1}$.

$Z$ is completely regular: Let $p \notin F$, closed in $A$. Then for some $\alpha, p \in T_{\alpha}^{*}$. Now $F \cap T_{\alpha}^{*}$ is closed in $T_{\alpha}^{*}$ which is completely regular so there exists a $g: T_{\alpha}^{*} \rightarrow[0,1]$ such that $g(p)=0$ and $g\left[F \cap T_{\alpha}^{*}\right]=1$. We extend $g$ to $g^{*}: Z \rightarrow$ $[0,1]$ by setting $g^{*}\left[Z-T_{\alpha}^{*}\right]=1$. Since $T_{\alpha}^{*}$ and $Z-T_{\alpha}^{*}$ are disjoint open-closed sets, $g^{*}$ is continuous.

We now define a canonical map $\phi: Z \stackrel{\text { onto }}{\longrightarrow} S\left(\omega_{1}\right)$ as follows: $\phi\left[T_{\gamma}^{*}-T_{\gamma}\right]=\gamma$. (Note that for isolated ordinals $\gamma$, this means that $\phi(\gamma)=\gamma$, if we retain the notation in $Z$ for isolated points.) To show $\phi$ is continuous, let $U$ be open in $S\left(\omega_{1}\right)$. We will show that $\phi^{-1}[U] \cap T_{\alpha}^{*}$ is open in $T_{\alpha}^{*}$ for every $\alpha$. Now, for a fixed $\alpha<\omega_{1}, \phi^{-1}[U] \cap T_{\alpha}^{*}=\phi^{-1}\left[U \cap S^{*}(\alpha)\right]$. Let $x$ be any element of $U \cap S^{*}(\alpha)$. If $x$ is isolated $\phi^{-1}(x)$ is isolated and hence open in $T_{\alpha}^{*}$. If $x \leqq \alpha$ is a limit ordinal, there is an isolated ordinal $\gamma<x$ such that $[\gamma, x+1] \subset U \cap S^{*}(\alpha)$. Then $\phi^{-1}[(\gamma, \mathrm{x}+1)]=T_{x}^{*}-T_{\gamma-1}^{*}$ which is open in $T_{x}^{*}$. But $T_{x}^{*}$ is open in $T_{\alpha}^{*}$ so $\phi^{-1}[(\gamma, x+1)]$ is open in $T_{\alpha}^{*}$. We conclude that $\phi^{-1}[U] \cap T_{\alpha}^{*}=\phi^{-1}\left[U \cap S^{*}(\alpha)\right]$ is a union of open sets in $T_{\alpha}$ and hence is open in $T_{\alpha}^{*}$. Since $Z$ is the topological sum of all the $T_{\alpha}^{*}, \phi$ is continuous.

$Z$ is $S\left(\omega_{1}\right)$-compact. To show this we use Mrowka's Theorem. Let $X=Z$, $E_{1}=S, E=S\left(\omega_{1}\right)$ and $Y=S\left(\omega_{1}\right) \cup\left\{\omega_{1}\right\}$. Then $\beta_{S\left(\omega_{1}\right)} Z$ is the set of all points 
in $\beta_{0} Z-X_{0}$ where $X_{0}$ is the set of all points $p_{0}$ of $\beta_{0} Z$ such that there is a $g: \beta_{D} Z \rightarrow \beta S\left(\omega_{1}\right)$ with $g(p) \in S\left(\omega_{1}\right)$ for $p \in Z$ and $g\left(p_{0}\right)=\omega_{1}$. To obtain such a $g$ we take the previously defined $\phi: Z \rightarrow S\left(\omega_{1}\right)$ and extend it to $\phi^{*}: \beta_{\mathbb{D}} Z \rightarrow$ $\beta S\left(\omega_{1}\right)$ by setting $\phi^{*}\left[\beta_{\mathcal{O}} Z-Z\right]=\omega_{1}$. Then $\phi^{*}$ is such a function $g$.

$S\left(\omega_{1}\right)$ is not $Z$-compact. For suppose it is; then $S\left(\omega_{1}\right) \subset_{c l} Z^{m}$ for some cardinal $m$. Now at least one of the projections must be infinite for otherwise $S\left(\omega_{1}\right)$ would be a closed subspace of a compact space. Thus we have a continuous $f: S\left(\omega_{1}\right) \rightarrow Z$ with infinitely many values. We assert that for some $\xi_{0}<\omega_{1}$, $f\left[0, \xi_{0}\right]$ is infinite. This follows from the following property from set theory: if $\left\{A_{\xi}: \xi<\omega_{1}\right\}$ is a non-decreasing sequence of finite sets, then $\cup\left\{A_{\xi}: \xi<\omega_{1}\right\}$ is finite. Therefore we have $f\left[0, \xi_{0}\right]$ compact and countably infinite in $Z$ which cannot be.

ReMARK. By the same procedures used in constructing the space $Z$, we can obtain spaces $Z_{2}, Z_{3}, \cdots$ such that

$$
K(\cong) \subset K\left(Z_{n}\right) \subset K\left(S\left(\omega_{n}\right)\right),
$$

where the containments are proper.

The space $Z$ in the preceding was constructed to show that the class $K\left(S\left(\omega_{1}\right)\right)$ is not an atom in the lattice of all classes of $E$-compactness. The natural question then is whether $K(Z)$ is an atom. We conclude this paper by constructing a space $X$ which will show that this is not true. The fact that there is nothing very special about this space $X$ leads the author to conjecture that there is no atom between $K(\supseteq)$ and $K\left(\mathrm{~S}\left(\omega_{1}\right)\right)$.

The space $X$. Consider the space $Z$ and from $T_{\omega_{0}}^{*}(=\beta N)$ delete one point

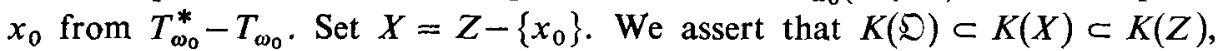
where again the containments are proper.

(i) We first show that $X$ is not $Z$-compact by showing that $X$ is not contained as a closed copy of any power of $Z$. Suppose this is false and that for some cardinal $m$ we have $X \subset_{c l} Z^{m}$. Now the subset $T_{\omega_{0}}^{*}-\left\{x_{0}\right\}$ is a closed subset of the space $X$ and hence it is closed in $Z^{m}$ also. However, since the subset $T_{\omega_{0}}$ of $X$ is countable, for each coordinate space $Z_{\xi}(=Z)$, the projection $\pi_{\xi}\left[T_{\omega_{0}}\right]$ upon $Z_{\xi}$ is contained in $T_{\alpha_{\xi}}^{*}$ for some limit ordinal $\alpha_{\xi}<\omega_{1}$. Since projections are continuous, we conclude that for each $\xi, \pi_{\xi}\left[T_{\omega_{0}}^{*}-\{x\}\right]=\pi_{\xi}\left[\mathrm{cl}_{\xi} T_{\omega_{0}}\right]$ is also contained in $T_{\alpha_{\xi}}^{*}$ since this set is closed in $Z_{\xi}$. Therefore we have $X$ is contained as a closed subset of the compact space $\prod_{\xi \in \Xi} T_{\alpha \xi}^{*}$ and hence is compact. This cannot be since for any $p \in \beta N-N, \beta N-\{p\}$ is not compact.

(ii) We show that $Z$ is $X$-compact by embedding $Z$ in a power of $X$. First note that $T_{\omega_{0}}^{*} \subset Z$ is open-closed and hemeomorphic to $\beta N$. Since $\beta N$ is $N$ compact, $T_{\omega_{0}}^{*} \subset_{c l} N^{m}=\left(T_{\omega_{0}}\right)^{m}$ for some cardinal $m$. For convenience let the subset $T_{\omega_{0}}^{*}-\left\{x_{0}\right\}$ of $X$ be denoted by $A$. We can then assume that $T_{\omega_{0}}^{*}$ has been embedded in $A^{m} \subset X^{m}$ by some homeomorphism, say $h$. But then $h\left[T_{\omega_{0}}^{*}\right]$ is a compact subset 
of the Hausdorff space $X^{m}$ and so is closed in $X^{m}$. It is a trivial matter to see that $Z-T_{\omega_{0}}^{*}$ is embeddable as a closed subset of $X^{m}$ which is disjoint from $h\left[T_{\omega_{0}}^{*}\right]$. Thus $Z \subset_{c l} X^{m}$.

\section{References}

[1] R. Engelking and S. Mrowka, 'On E-compact Spaces', Bull. Acad. Polon Sci. Cl. III 6 (1958), $429-436$.

[2] S. Mrowka, 'Further results on E-compact spaces. I' Acta Mathematics, 120 (1968), 161-185.

[3] J. G. Hocking and G. S. Young, Topology (Addison-Wesley, Reading Mass., 1961).

[4] L. Gillman and M. Jerison, Rings of Continuous Functions (D. Van Nostrand, New York, 1960).

[5] W. Sierpinski, Cardinal and Ordinal Numbers (Warsaw, 1958).

[6] H. Bachman, Transfinite Zahlen (Berlin, 1955.)

[7] A. Abian, 'On the Confinality of Ordinal Numbers', Math. Ann. 179 (1969), 142-152. 